\title{
Piezoelectricity of a Ferrocene-based Organic Small Molecule
}

Ling Li,,${ }^{[a] \#}$ Jianyu Zhang, ${ }^{[b] \#}$ Xiaohui Wang, ${ }^{[a]}$ Qingsong Wang, ${ }^{[a]}$ Wei Gao, ${ }^{[\mathrm{d}]}$ Xing Feng, ${ }^{*[\mathrm{a}]}$ Carl Redshaw, ${ }^{[\mathrm{c}]}$ Nengjie Huo, ${ }^{[\mathrm{d}]}$ Jingbo Li, ${ }^{*[\mathrm{a}, \mathrm{d}]}$ Jacky W. Y. Lam, ${ }^{[\mathrm{b}]}$ Ben Zhong Tang*[b]

[a] L. Ling, X. Wang, Q. Wang, Dr. X. Feng, Prof. Dr. J. B. Li

Guangdong Provincial Key Laboratory of Functional Soft Condensed Matter, School of Material and Energy, Guangdong University of Technology, Guangzhou 510006, P.

R. China

E-mail: hyxhn@sina.com (X. Feng)

[b] J. Zhang, Prof. J. W. Y. Lam, Prof. Dr. B. Z. Tang

Department of Chemistry, The Hong Kong Branch of Chinese National Engineering Research Center for Tissue Restoration and Reconstruction, Institute for Advanced Study, Department of Chemical and Biological Engineering, Division of Life Science, State Key Laboratory of Molecular Neuroscience, The Hong Kong University of Science and Technology, Clear Water Bay, Kowloon, Hong Kong, China

E-mail: tangbenz@ust.hk (B. Z. Tang)

[c] Prof. Dr. C. Redshaw

Department of Chemistry, University of Hull, Cottingham Road, Hull, Yorkshire HU6 7RX, UK.

[d] Dr. W. Gao, Dr. N. Huo, Prof. Dr. J. B. Li

Institute of Semiconductors, South China Normal University, Guangzhou 510631, P. R. China.

E-mail: jbli@ semi.ac.cn

Abstract: Non-centrosymmetric crystals with piezoelectric properties have emerged as promising materials for smart wearable systems and biomimetic robots. Here we present a novel small ferrocene-based organic molecule crystal $(\mathbf{F c}-\mathbf{C z})$ possessing high anisotropic-dependent optical and electronic properties, which has been utilized as an ultrasensitive piezoelectric material for the development of a strain sensor. The flexible piezoelectric sensor can distinguish subtle strain or deformations (such as wrist motion) with fast response time $(<40 \mathrm{~ms})$ via detectable piezoelectric signals $\left(I_{\max }=580 \mathrm{pA}\right)$. Density functional theory (DFT) indicated that the external pressure can affect the dipole moment by changing the molecular configuration of the asymmetric single crystal Fc-Cz in the crystalline state, leading to a change of polarity, as well as an enhanced dielectric constant. Based on our knowledge, this work is the first example verifying that artificial organic small molecules can serve as simple, stable, high- 
performance tactile sensors, and this has the potential to open the door to low-cost flexible wearable devices and energy harvesting applications.

\section{Introduction}

The development of advanced piezoelectric materials for tactile electronic skin (e-skin) has attracted recent attention, and this has resulted in remarkable applications, for example as wearable devices and in smart robotics. ${ }^{[1-4]}$ Piezoelectricity is an inherent property of non-centrosymmetric crystals, and is highly sensitive and can distinguish subtle changes in the external environment (such as temperature and pressure), and generates a corresponding electrical signal. $\left.{ }^{[5} 6\right]$ The underlying mechanism of piezotronic effect is the polarization charges of piezoelectric materials which are generated under a mechanical deformation along the polarization orientation in the noncentrosymmetric materials. ${ }^{[7]}$ To-date, the engineering of piezoelectric materials with a view to e-skin technology has focused on the use of carbon-based materials (carbon nanotubes $^{[8]}$, graphene ${ }^{[9]}$ ), polymer nanofibers ${ }^{[10]}$, perovskite ${ }^{[11]}$, 2D layered materials

(such as $\left.\alpha-\mathrm{In}_{2} \mathrm{Se}_{3}\right)^{[12-14]}$, and inorganic complexes ${ }^{[15-16]}$. However, few examples have exploited small organic molecules to fabricate flexible wearable electronic devices at the nanoscale level. In comparison to inorganic piezoelectrics, artificial small organic molecules are potentially better piezoelectric materials because i) they possess varied and tunable molecular frameworks, ii) they can adopt precise molecular structures possessing unique molecular conformations, and iii) they exhibit controllable molecular packing in the solid state. Moreover, theoretical calculations have revealed that the presence of hydrogen bonding plays a significant role in achieving a larger maximum piezo-response. ${ }^{[17]}$ Indeed, organic materials with numerous hydrogen bonds in their molecular packing can potentially be efficient organic piezomaterials for strengthening the piezoelectric properties.

It is found that bio-organic and natural biomaterials possess weak piezoelectric effects, whilst breakthrough discoveries revealed that amino acids/proteins can be used as piezoelectric materials for nanoelectronic applications. ${ }^{[18-19]}$ Thompson and 
coworkers utilized the piezoelectric properties of glycine polymorphs for potential application in biopiezoelectric sensing and energy harvesting ${ }^{[20]}$. Interestingly, Kholkin et al. observed that self-assembled diphenylalanine peptide nanotubes (PNTs) exhibited a strong anisotropy-dependent piezoelectricity with the orientation of polarization along the axis of the tube ${ }^{[21]}$. However, currently, the utilization of unique molecular architectures with strong piezoelectricity based on artificial organic molecules remains a challenge.

Ferrocene, $\mathrm{Fe}\left(\mathrm{C}_{5} \mathrm{H}_{5}\right)_{2}$, is an aromatic hydrocarbon compound with a sandwiched structure, and the Fe atom between the two parallel cyclopentadienyl $(\mathrm{Cp})$ rings exhibits an intriguing magnetic property and shows promise for utilization in electron transport and spin filtering. ${ }^{[22-23]}$ Thus, various ferrocene-based complexes as organic photoelectric-magnetic functional materials have been thoroughly investigated by experimental and theoretical methods. On the other hand, due to the high-energy lone pair at the $\mathrm{Fe}$ atom in the ferrocene unit, ferrocene-containing compounds prefer to exhibit weak/non-emission through intramolecular photoinduced electron transfer (PET) reactions, which could be an excellent "turn-on" probe to detect the metal cation and anion by lowing the energy of the lone pair. ${ }^{[24]}$

In 2001, Tang's group observed an abnormal photophysical phenomenon and defined it as aggregation-induced emission (AIE), whereby a series of twisted luminogens exhibited non-luminescent in solution but the fluorescence intensity was enhanced in the solid state. ${ }^{[25-27]}$ Cyanostilbene derivatives are widely used in developing new AIE luminogens, and this category of organic molecules in the solid state exhibited mechanoluminescence (ML) and high luminescence photoluminescence (PL) efficiency, and the conformation of the packing mode plays a significant role in affecting the ML behavior. ${ }^{[28-30]}$ Thus, what would happen when the AIE luminogens meet the organic photoelectric-magnetic materials?

In this work, by combining the cyanostilbene and ferrocene motifs, a new small organic molecule (Fc-Cz) was readily designed and synthesized by a Knoevenagel reaction, and its crystal packing, thermal behavior, photophysical properties, and electrical properties have been fully investigated. Crystalline $\mathbf{F c}-\mathbf{C z}$ illustrated strong 
anisotropy-dependent piezoelectricity under external stimuli with a maximum peak current up to $580 \mathrm{pA}$. Moreover, the advanced piezoelectric property of $\mathbf{F c}-\mathbf{C z}$ was used to fabricate a flexible tactile sensor for recording human activity (hand movements) with highly sensitivity.

\section{Results and Discussion}

\section{Synthesis and General Properties}

A Knoevenagel reaction between ferrocene carboxaldehyde and 2-(4'-(8a,9a-dihydro9H-carbazol-9-yl)-[1,10-biphenyl]-4-

yl)acetonitrile (1) ${ }^{[31]}$ in dry ethanol afforded the target compound (Fc-Cz) in $80 \%$ yield; the synthetic route is illustrated in Scheme 1. The molecular structure $\mathbf{F c}-\mathbf{C z}$ was fully characterized by ${ }^{1} \mathrm{H} /{ }^{13} \mathrm{C}$ NMR spectra, high resolution mass spectra (HRMS) and single crystal X-ray crystallography. Fc-Cz possesses excellent solubility in common organic solvents, such as toluene, tetrahydrofuran (THF), dichloromethane, dimethyl sulfoxide (DMSO) and $N, N$-dimethylformamide (DMF).

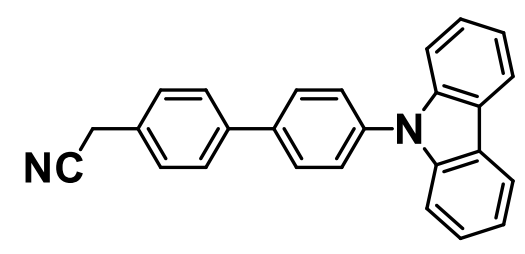

1

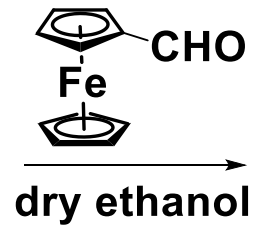

dry ethanol

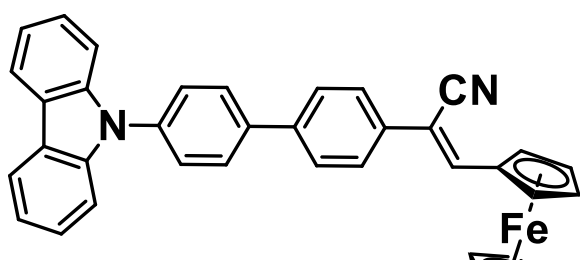

Fc-Cz

Scheme 1. Synthetic route to compound Fc-Cz.

\section{Single crystal X-ray diffraction analysis}

A single crystal of compound $\mathbf{F c}-\mathbf{C z}$ was cultivated by slow evaporation of a mixture of hexane and chloroform, and the detailed molecular structure and crystal pattern were confirmed by X-ray crystallography and are illustrated in Figure 1. The basic crystallographic data and structure refinement parameters are summarized in Table S1. For crystalline $\mathbf{F c}-\mathbf{C z}$, the asymmetric unit contains eight molecules, the molecules adopt a twisted conformation between the carbazole and two phenyl moieties with the 
twist angles of $61.73^{\circ}$ and $78.10^{\circ}$, respectively. The ferrocene unit is almost parallel with the carbazole unit, and the compound $\mathbf{F c}-\mathbf{C z}$ adopts a head-to-tail packing pattern through several C-H $\cdots \pi$ interactions with distances in the range of $2.944-3.031 \AA$. The crystals formed an ordered tight crystal-state packing along the $a$-axis and $c$-axis directed by weak $\mathrm{C}-\mathrm{H} \cdots \pi$ interactions, but adopted a loose packing pattern with unconspicuous overlap and weak intermolecular interactions along the $b$-axis. ${ }^{[32]}$ Moreover, it is interesting that $\mathbf{F c}-\mathbf{C z}$ adopts a herringbone pattern along the $b$-axis (Figure S3-2), and this packing would contribute to improving the mechanical properties of the crystal. ${ }^{[33]}$

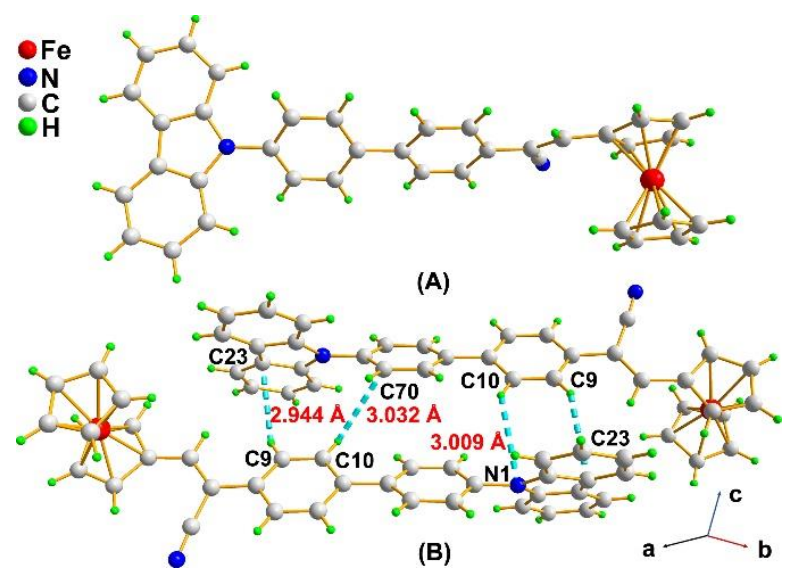

Figure 1. A) Crystal structure of compound $\mathbf{F c}-\mathbf{C z}$, B) Packing structure adopted via C$\mathrm{H} \pi$ interactions.

\section{UV-vis measurement}

The UV-visible absorption spectra of compound $\mathbf{F c}-\mathbf{C z}$ were measured in THF solution $\left(10^{-5} \mathrm{M}\right)$ and in the solid state. As shown in Figure S4-1, the UV spectra exhibited two strong absorption peaks at $278 \mathrm{~nm}$ and $341 \mathrm{~nm}$, respectively, and a weak absorption band in the range of 430-600 nm, which was assigned to the metal-to-ligand charge transfer (MLCT) transition. The compound Fc-Cz exhibited a maximum emission peak at $397 \mathrm{~nm}$ in solution, which may originate from the phenyl-phenyl-carbazole units. Although the crystal Fc-Cz did not emit any fluorescene in solid state using a fluorescence spectrophotometry $\left(\lambda_{\mathrm{ex}}=291 \mathrm{~nm}\right)$, a very weak, wide emission peak in range 350 to $600 \mathrm{~nm}$ was observed under $325 \mathrm{~nm}$ laser irradiation (Figure S4). This 
maybe due to the synergic effects of the strong twisted intramolecular charge transfer (TICT) effect and the strong PET from the ferrocene to the phenyl-phenyl carbazole fragment.

\section{Polarization-dependent photoluminescence (PL) spectrum}

The molecule Fc-Cz crystallizes in the monoclinic $P 2{ }_{1} / \mathrm{c}$ space group, and its low symmetry in the unit cell indicated that the anisotropic excitons would dominate the excitonic state and enhance the excitonic effects. ${ }^{[34-35]}$ Thus, polarization-dependent photoluminescence (PL) was performed in order to understand the anisotropic optical properties via angle-resolved polarized photoluminescence spectroscopy (ARPPS). ${ }^{[36]}$ A high quality, single rod-like crystal $\mathbf{F c}-\mathbf{C z}(15 \times 50 \mu \mathrm{m})$ was transfered to the $\mathrm{SiO}_{2} / \mathrm{Si}$ substrate, the orientation-dependent photoluminescence property was measured by a $532 \mathrm{~nm}$ excitation laser and the PL diagram is illustrated in Figure 2. During the measurements, the rotation angle $(\theta)$ from $0^{\circ}$ to $360^{\circ}$ (every $10^{\circ}$ ) of the sample is operated in a clockwise direction.
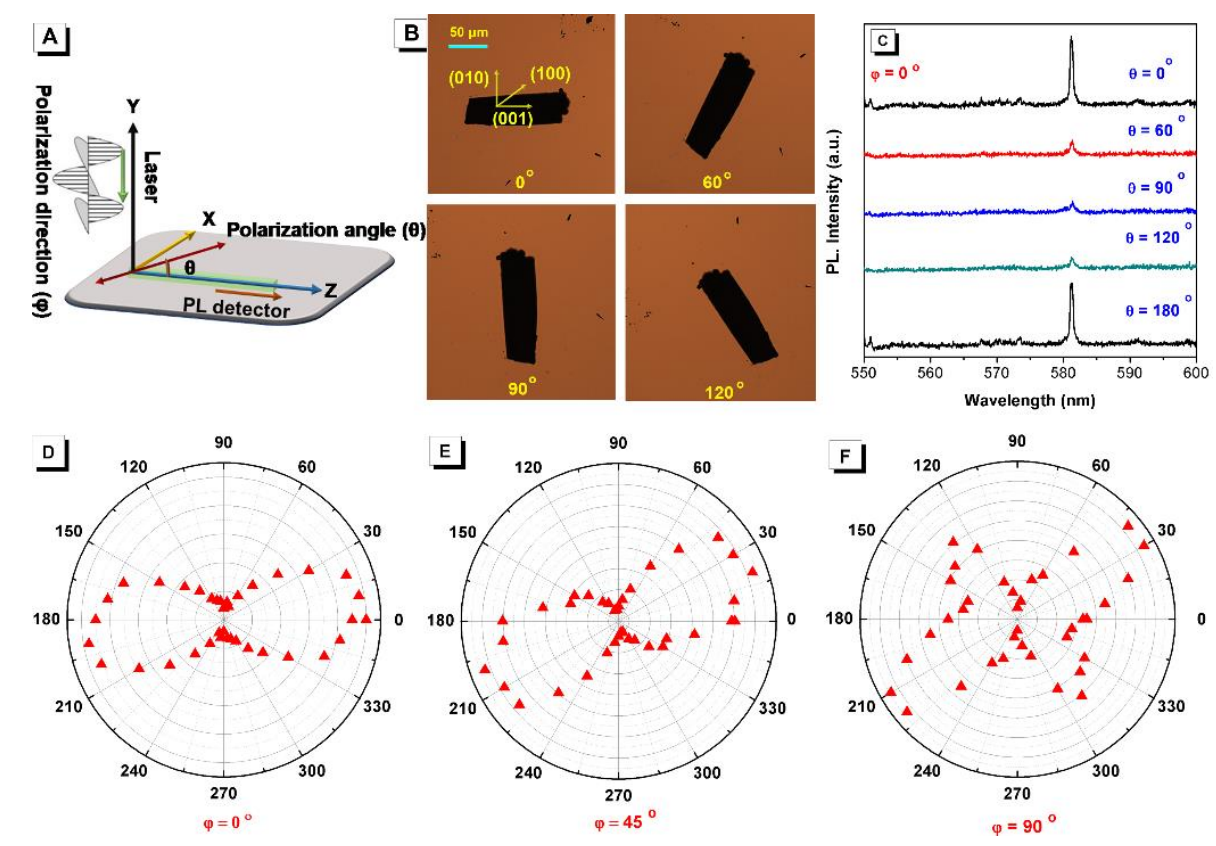

Figure 2. (A) Schematic of the PL measurement configuration. (B) Photograph of crystal $\mathbf{F c}-\mathbf{C z}$ on the the $\mathrm{Si} / \mathrm{SiO}_{2}$ substrate with polarization orientations. (C) PL spectra of different polarization angles. (D), (E) and (F) (E) and (F) Angular-dependent PL of the maximum intensity of peak at $583 \mathrm{~nm}$ 
In particular, a strong and intensive PL peak at $581 \mathrm{~nm}$ was observed under unpolarization. When the excitation laser polarization direction (between the incident light and PL detector: $\varphi$ ) is $0^{\circ}$, as the rotation angle $(\theta)$ changed from $0^{\circ}$ to $180^{\circ}$ (along the $z$-axis), the PL intensity generally decreased from 458 au to 76 au $\left(\theta=0^{\circ} \sim 90^{\circ}\right)$, then enhanced to 409 au $\left(\theta=90^{\circ} \sim 180^{\circ}\right)$. The maximum/minimum related intensity ratio $\left(I_{\max } / I_{\min }\right)$ was up to 6.0 and the polarization ratio $\rho_{\mathrm{PL}}=\left(I_{\max }-I_{\min }\right) /\left(I_{\max }+I_{\min }\right)$ was ca. 0.72. Clearly, the ARPPS indicated that the anisotropy showed a significant effect on the PL intensity with a two-lobed polar plot. When the excitation laser polarized along the $\varphi=45^{\circ}$ direction, the crystal $\mathbf{F c}-\mathbf{C z}$ exhibited a similar polarization PL behavior with a polarization ratio $\rho_{\mathrm{PL}}$ of 0.63 . It is interesting that the polar plot exhibited a four-leaf clover structure when the polarization orientation of excitation laser was $\varphi=90^{\circ}$. Generally, the polarization PL intensity was related to the angledependant near-gap excitons, ${ }^{[34-35]}$ thus, the polar plot indicated that the direct recombination of excitons was mainly concentrated along the $z$-axis (001 plane) of crystal Fc-Cz. For comparison, the above-mentioned polarization resolved spectra with $\varphi=0^{\circ}$ was repeatedly measured for similar polarization PL behavior at selected points (B and C) of this crystal sample, indicating that the same molecular orientation exists in the crystal of Fc-Cz along the $z$-axis (001) planes (Figure S4-6). As for the above ARPPS results, the crystal Fc-Cz exhibited well-defined anisotropy-dependent optical properties.

\section{Piezoelectric properties}

The fundamental properties of transport behavior (such as charge carrier mobilityes, anisotropic transport) for organic meterials are related to the development of highperformance and low-cost organic semiconducting devices. ${ }^{[37]}$ In our case, a flexible piezoelectric sensor was fabricated in a metal-organic semiconductor-metal (MOM) structure on the polyimide (PI) substrate, using silver pulp as metal electrodes, where the rod-like crystal Fc-Cz was fixed by a silver pulp along the $z$-axis ((001) plane); the channel length of the pressure sensor device was about ca. $25 \mu \mathrm{m}$ (Figure S7-1). Notably, the non-centrosymmetric monoclinic crystal Fc-Cz showed an obvious and stable piezoelectric effect, and even the PI substrate was bent under a periodic strain 
(bending-released motion) within a hundred cycles (Figure S6-3).
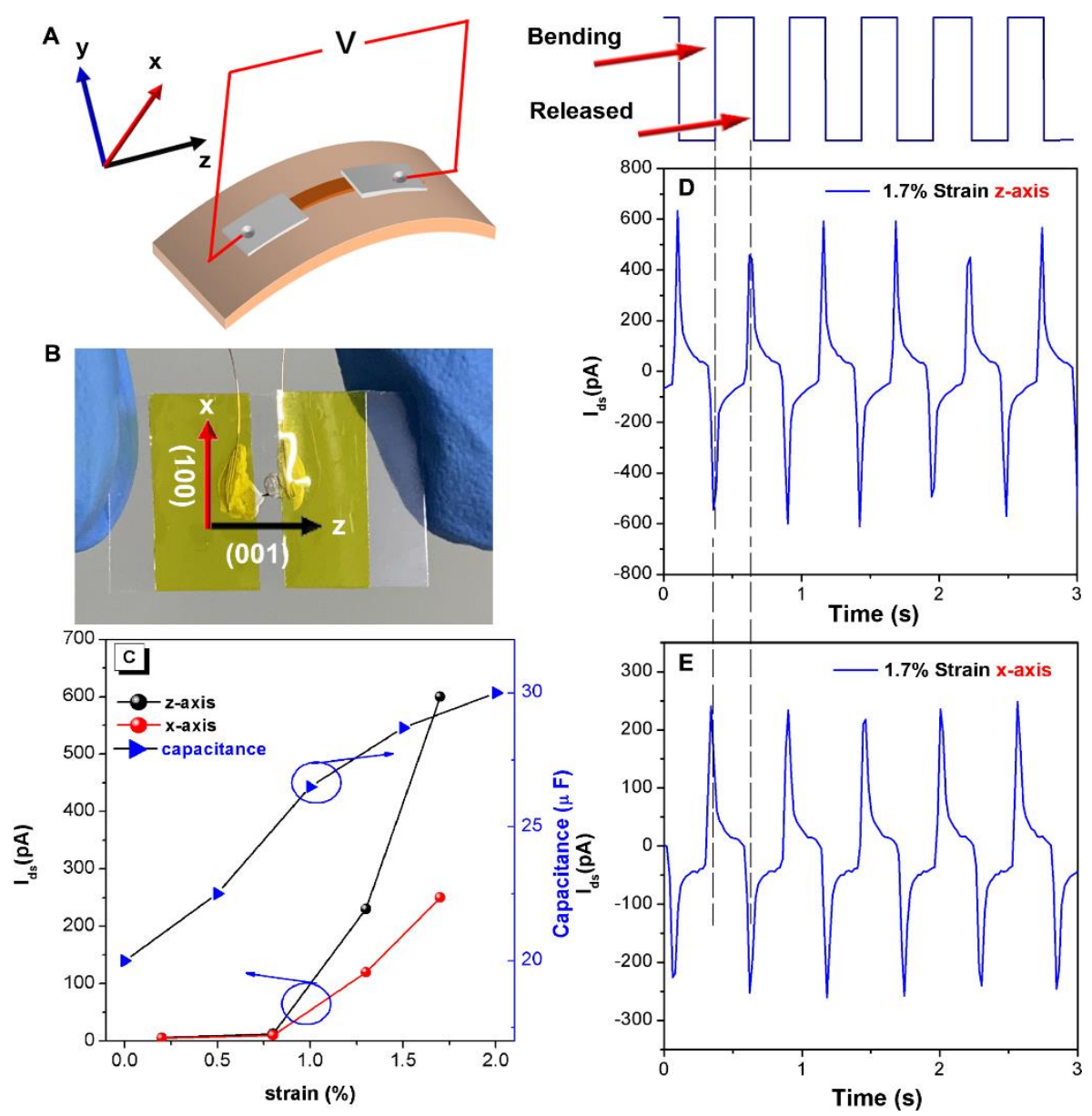

Figure 3. Piezoelectric outputs from the crystal of the ferrocene-based organic small molecule nanogenerators. (A) the sketch of the flexible piezoelectric nanogenerators. (B) the photograph of the flexible piezoelectric nanogenerators. (C) the relationship between Electrical output (capacitance) and strain. (D) and (E) Short-circuit current response of the ferrocene-based organic small molecule nanogenerator under periodic strain $(1.7 \%)$ along the $z$ - and $x$-axes.

The applied strain can be calculated by the following formula(1):

$$
\varepsilon=\mathrm{d} \sin \theta / \mathrm{L}
$$

where, $\varepsilon$ is the strain, $\mathrm{d}$ is the thickness of the substrate, $\theta$ is the angle between the tangent line along the end of the substrate and the horizontal line, $L$ is the length of the PL substrate. ${ }^{[7]}$

When the strain was applied to the device along the $z$-axis, a negative current output 
rapidly appeared within $40 \mathrm{~ms}$, and a positive output was observed within $<40 \mathrm{~ms}$ as the strain was removed. Figure 3 and Figure S6 reveal the dynamic piezoelectric signals as a function of strain from different directions. The dynamic piezoelectric signals were enhanced as the applied strain increased from $0.5 \%$ to $1.7 \%$ along the $z$-axis $(001)$ (Figure 3c and Figure S6-2). However, under the same strain, the maximum peak current was ca. 2 -fold that along the $z$-axis (001) compared to $x$-axis (100), due to the anisotropy of the crystal $\mathbf{F c}-\mathbf{C z}$, which was agreement with previous reports on 2D layered materials ${ }^{[38]}$. In particular, when the applied strain was up to $1.7 \%$ along the $x$ axis, the maximum peak current was more than $580 \mathrm{pA}$, which was higher (ca. 80-fold) than under $0.5 \%$ strain. This value is larger than that reported previously for piezoelectric materials, such as two-dimensional (2D) ultrathin semiconductors (GaS, GaSe, $\operatorname{In}_{2} \mathrm{Se}_{3}, \mathrm{MoS}_{2}, \mathrm{MoSe}_{2}$, graphene-based composite $\left.{ }^{[14,15,39-41]}\right)$, indicating that the Fc-Cz crystal based flexible piezoelectric device was ultrasensitive to external pressure. For comparison, the piezoelectricity properties of the compound $2-\left(4^{\prime}-(8 a, 9 a-\right.$ dihydro-9H-carbazol-9-yl)-[1,10-biphenyl]-4-yl)acetonitrile (1) was also investigated, and this also displayed a detectable electronic signal under extra strain. However, in the absence of the ferrocene units, compound $\mathbf{1}$ exhibited brittleness with a low peak current ( $\sim 38 \mathrm{pA})$ under $1.3 \%$ strain (Figure S6-5). Thus, these results suggest that our ferrocene-based organic small molecular semiconductor materials/flexible piezoelectric nanogenerators would be applicable for applications such as e-skin, selfpowered systems and as wearable sensors.

Furthermore, due to the ultrasensitivity toward pressure, the crystal $\mathbf{F c}-\mathbf{C z}$ was transferred to a PDMS substrate in order to investigate its potential application as a wearable sensor for the monitoring of human motion. As shown in Figure 4, the tactile sensor was fixed above the forefinger and encapsulated by a polyimide film. When the finger wrist adopted a periodic bending-stretching motion, the corresponding negative and positive electronic signals were observed, and along with the increasing of the bending angle, the piezoelectric current also increased. The maximum current was up to $3600 \mathrm{pA}$ when the finger bending angle was about $70^{\circ}$. The tactile sensor exhibited good stability, high sensitivity, and an excellent repetition for the recognition of the 
continuous movements of the human hand, and the entire measurement was recorded simultaneously on video (see Movie 1 in SI). Accordingly, such small molecular organic semiconductors like $\mathbf{F c}-\mathbf{C z}$ can be considered as a new generation of materials for use as wearable devices for practical implementation.

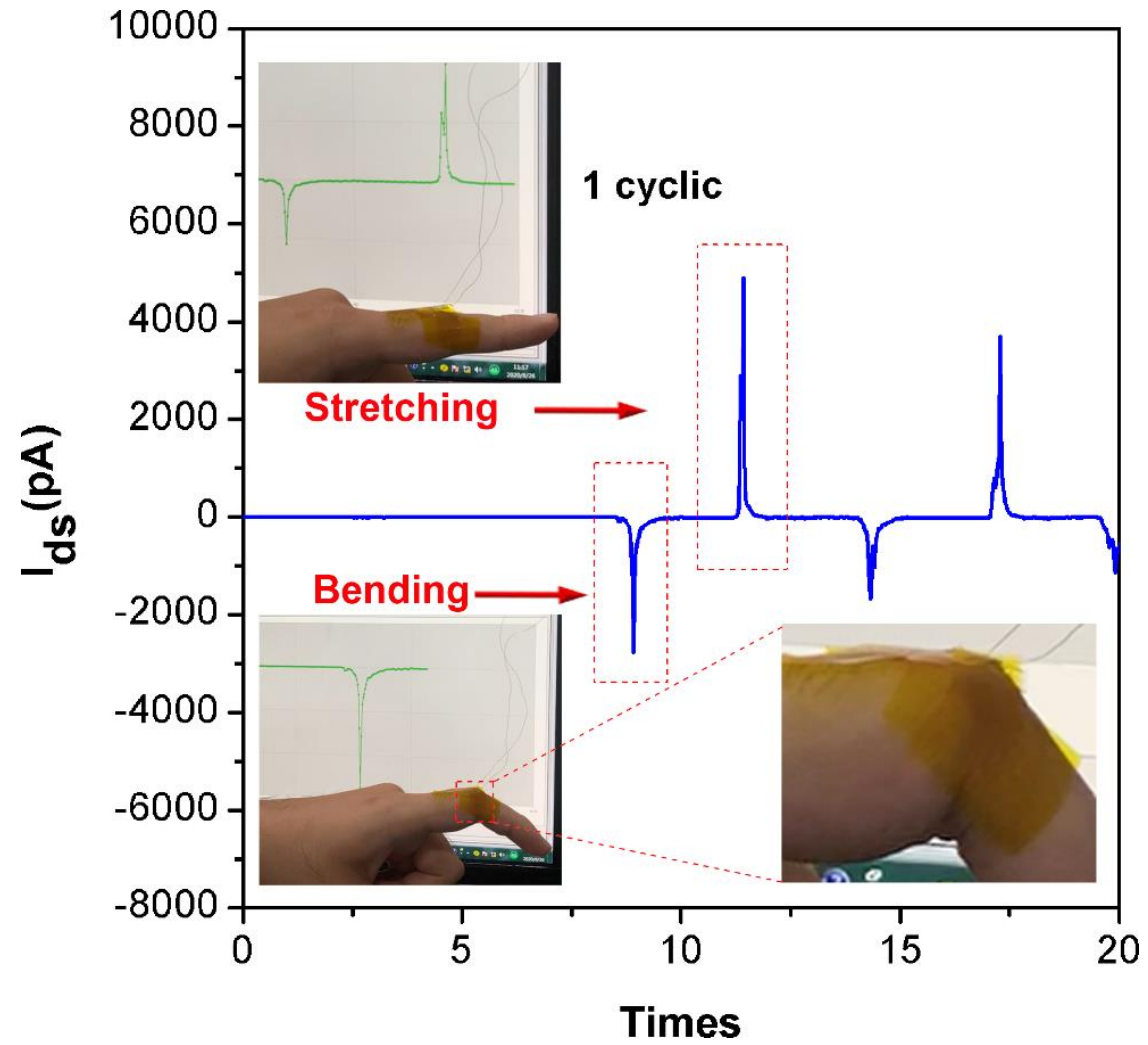

Figure 4. The flexible wearable device fixed on the forefinger and encapsulated by polyimide film substrate for the detection of pulse waves. Insert: the wrist wearing experiments under stretching-bending motion states.

\section{The relationship between piezoelectricity, dielectric and polarizability}

For organic semiconductor materials, the external force plays a significant role in the flexible organic molecular pattern in the crystal/aggregation state ${ }^{[42]}$, leading to certain types of macroscopic photophysical/chemical behavior, such as mechanoluminescence. ${ }^{[43-45]}$ Furthermore, the capacitance of the the flexible piezoelectric nanogenerator was measured, which was enhanced from 6 to $30 \mu \mathrm{F}$ as the strain increasing along the $x$-axis, which was agreement with the observed dynamic piezoelectric signals trends. The relationship between capacitance and dielectric 
constant can be illustrated by the formula (2):

$$
\varepsilon_{r}=\mathrm{C} \cdot \mathrm{d} / \varepsilon_{0} \mathrm{~S}
$$

where the $\varepsilon_{r}$ is the relative dielectric constant, $\mathrm{C}$ is the capacitance, $\varepsilon_{0}$ is the vacuum dielectric constant $\left(8.854 \times 10^{-12} \mathrm{~F} / \mathrm{m}\right)$, and $\mathrm{S}$ is the area of sample.

According to the empircal formula (3):

$$
\mathrm{X}=\varepsilon_{r}-\varepsilon_{0}
$$

where $X$ is the polarizability, and $\varepsilon_{0}$ is the vacuum dielectric constant.

Thus, the formula can be changed to (4)

$$
\mathrm{X}=\mathrm{C} \cdot \mathrm{d} /\left(\varepsilon_{0} \cdot \mathrm{S}\right)-\varepsilon_{0}
$$

According to the formula, the polarizability is positively associated with the relative dielectric constant. We inferred that the relative polarizability, as well as dipole moment of the crystal $\mathbf{F c}-\mathbf{C z}$ also would be enhanced with increased strain force. ${ }^{[46]}$ To further understand the relationship between piezoelectricity, dielectric and polarizability of the small molecular organic semiconductor $\mathbf{F c}-\mathbf{C z}$, density functional theory (DFT) methods were used at the PBE0/ def2-SVP level. Analytical frequency calculations were also performed at the same level of theory to confirm that the optimized structures were at the minimum point. Clearly, the highest occupied molecular orbital (HOMO) and the lowest unoccupied molecular orbital (LUMO) were isolated and were mainly located on the carbazole unit and the diphenyl fragment, respectively. The calculated HOMO and LUMO energies were about $-5.93 \mathrm{eV}$ and $-2.20 \mathrm{eV}$, respectively, and the energy gap between them was $3.73 \mathrm{eV}$ (Figure 5A). Furthermore, the HOMO level of compound Fc-Cz was also confirmed by XPS with $-5.72 \mathrm{eV}$. The optical energy gap $(3.14 \mathrm{eV})$ derived from the lowest energy absorption onset in the absorption spectra was $3.14 \mathrm{eV}$, and therefore the corresponding LUMO level was $-2.58 \mathrm{eV}$. The above results revealed good agreement between the experimental and theoretical studies. 

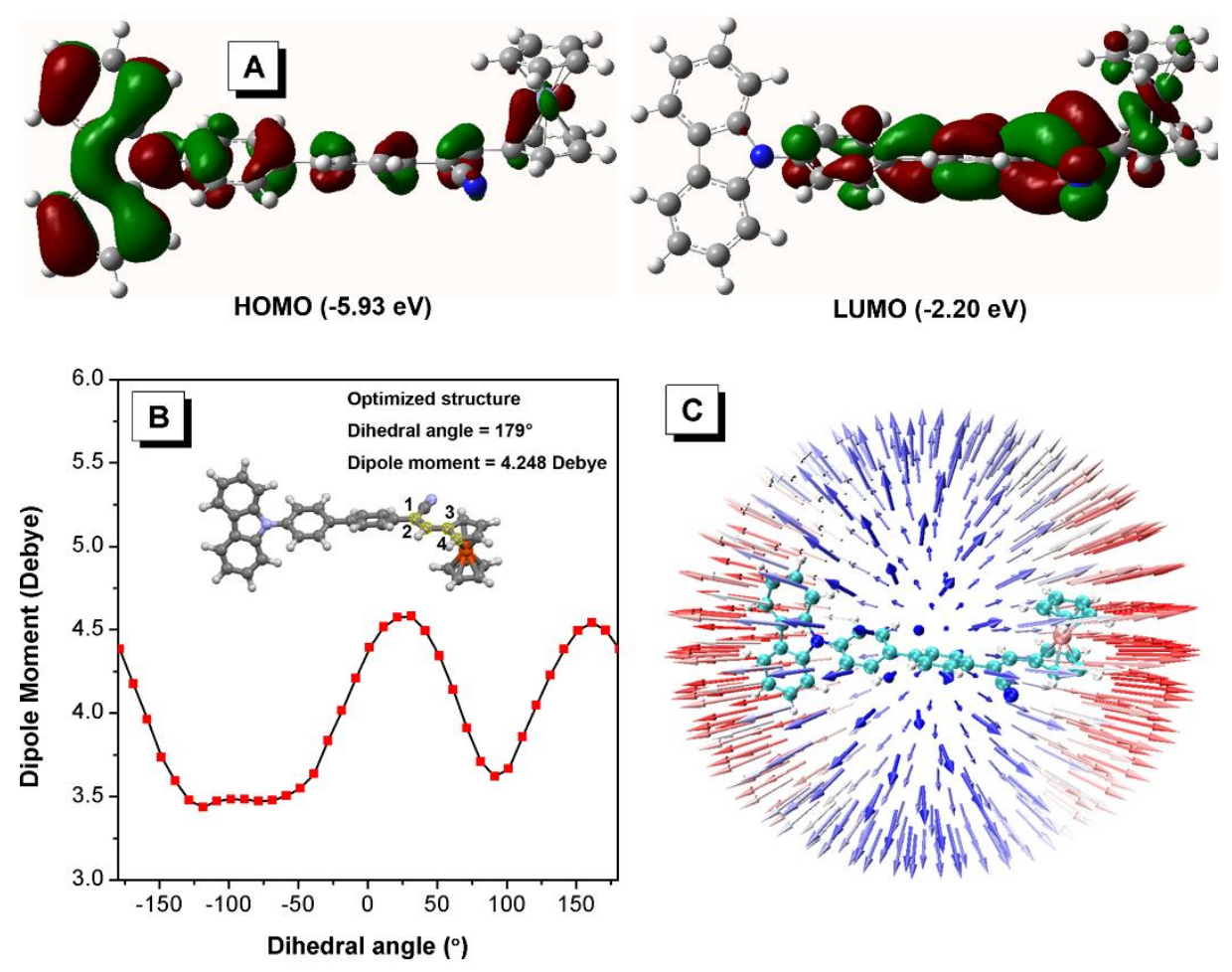

Figure 5. (A) Computed molecular orbital plots of compound Fc-Cz. (B) Calculated dipole moment of $\mathbf{F c}-\mathbf{C z}$ with different dihedral angles ( $\angle 1-2-3-4)$. (C) Visualization of the polarizability tensor of Fc-Cz. The unit sphere representation is colored with red being the largest magnitude effective dipoles and blue being the smallest magnitude effective dipoles, calculated at PBE0/def2-SVPD level, Gaussian 16 program.

Based on the optimized structure, different structures were scanned along the ferrocene group (dihedral angle $\angle 1-2-3-4$, as shown in Figure $5 B$ ) at the same level, Their dipole moments were further evaluated at the PBE0/def2-SVPD level. Based on optimized structural conformation, the polarizability and the surface of the polarizability tensor were analyzed using the Multiwfn package and VMD software. ${ }^{[47-}$ 48] The ferrocene unit was almost perpendicular to the phenyl ring at an angle of 71.7 ${ }^{\circ}$ in the optimized structure (the dihedral angle $\angle 1-2-3-4=179^{\circ}$ ); the corresponding dipole moment was 4.248 Debye. Indeed, as the dihedral angle $\angle 1-2-3-4$ changed in the range from $0^{\circ} 180^{\circ}$ to $90^{\circ}$ (or from $-180^{\circ}$ to $-120^{\circ}$ ), the dipole moment decreased from 4.58 to 3.62 (or 3.50) Debye. The comparatively large change of dipole with dihedral angle (large slope) indicated that small changes of molecular comformation 
can greatly affect the molecular dipole moment. Thus, we inferred that the periodic strain within the Fc-Cz device would make the molecular configuration change slightly, thereby leading to a relatively dynamic dipole moment fluctuation, which would favor variational polarizability. Furthermore, the 3D visualization of the first hyperpolarizability tensor was estimated to obtain insight into the relationship between the piezoelectric properties and the molecular structure. ${ }^{[47]}$ The visualization unit sphere indicated that the maximum second harmonic was along the $z$-axis of the molecular framework compared to the vertical direction (y-axis), which corresponded to the (010) planes of the crystal Fc-Cz. ${ }^{[49]}$ On the other hand, Hirshfeld surface analysis was performed to quantify the intermolecular interaction in the crystal state, which provides a relative proportion of $\mathrm{C} \cdots \mathrm{H}, \mathrm{N} \cdots \mathrm{H}$ and $\mathrm{H} \cdots \mathrm{H}$ with intermolecular contacts at $38.9 \%, 8.0 \%$ and $50.8 \%$, respectively. The presence of weak intermolecular interactions contributes to the enhancement of the piezo-coefficient of the designed organic piezoelectric material Fc-Cz.

\section{Conclusion}

Piezoelectric materials, as emerging advanced materials, have potential application in energy harvesting, microelectronics, and tactile e-skin. This article presents the high anisotropy-piezoelectric properties $(\sim 580 \mathrm{pA})$ and fast response $(<40 \mathrm{~ms})$ of a small organic molecular crystal (Fc-Cz). Due to its low symmetry, the crystal displayed orientation-dependent photoluminescence properties with a maximum polarity ratio of 0.72. The experimental and theoretical studies indicated that there exists a positive correlation between piezoelectricity, dielectric and polarizability, where higher external pressure would achieve larger dynamic piezoelectric signals. Furthermore, a tactile sensor device based on crystalline Fc-Cz exhibited great stability, high sensitivity, and great reproducibility when recognizing the continuous movements of a human hand. This work has pioneered the use of such artificial organic materials as piezoelectric materials for potential application in e-skin, which will open up the possibility of using other small organic molecular materials for a range of application in wearable devices, energy harvesting, and human-machine interaction. 


\section{Acknowledgements}

L. Ling and J. Zhang contributed equally to this work. This work was supported by the National Natural Science Foundation of China (21975054 and 21602014), Natural Science Foundation of Guangdong Province of China (2019A1515010925), Guangdong Province Training Program of Innovation and Entrepreneurship for Undergraduates, "One Hundred Talents Program" of the Guangdong University of Technology (GDUT) (1108-220413205), Guangdong provincial key laboratory of functional soft condensed matter of the Guangdong University of Technology (GDUT) (220413205). CR thanks the EPSRC for an Overseas Travel Grant (EP/R023816/1).

Keywords: piezoelectricity • aggregation-induced emission • organic small molecules - polarizability $\bullet$ wearable devices

[1] A. M. Manjón-Sanz, M. R. Dolgos, Chem. Mater. 2018, 30, 8718-8726.

[2] J. Rao, Z. Chen, D. Zhao, R. Ma, W. Yi, C. Zhang, D. Liu, X. Chen, Y. Yang, X. Wang, J. Wang, Y. Yin, X. Wang, G. Yang, F. Yi, Nano Energy. 2020, 75, 105073.

[3] J. Wu, D. Xiao, J. Zhu, Chem. Rev. 2015, 115, 2559-2595.

[4] H. Zhou, Y. Zhang, Y. Qiu, H. Wu, W. Qin, Y. Liao, Q. Yu, H. Cheng, Biosens. Bioelectron. 2020, 168, 112569

[5] B. Y. Lee, J. Zhang, C. Zueger, W.-J. Chung, S. Yoo, E. Wang, J. Meyer, R. Ramesh, S.-W. Lee, Nat. Nanotechnol. 2012, 7, 351-356.

[6] Z. L. Wang, J. H. Song, Science. 2006, 312, 242-246.

[7] L. Li, W. Gao, H. Chen, K. Zhao, P. Wen, Y. Yang, X. Wang, Z. Wei, N. Huo, J. Li, Adv. Electron. Mater. 2020, 6, 1901441.

[8] F. Mokhtari, Z. Cheng, R. Raad, J. Xi, J. Foroughi, J. Mater. Chem. A. 2020, 8, 9496-9522.

[9] M. T. Ong, E. J. Reed, ACS Nano. 2012, 6, 1387-1394.

[10] P. Snapp, C. Cho, D. Lee, M. F. Haque, S. Nam, C. Park, Adv. Mater. 2020, 32, 2004607.

[11] J. Hao, W. Li, J. Zhai, H. Chen, Mater. Sci. Eng. R Rep. 2019, 135, 1-57.

[12] C. Cui, F. Xue, W.-J. Hu, L.-J. Li, NPJ. 2D. Mater. Appl. 2018, 2, 24.

[13] S. A. Han, J. Lee, J. Lin, S.-W. Kim, J. H. Kim, Nano Energy. 2019, 57, 680-691.

[14] F. Xue, J. Zhang, W. Hu, W.-T. Hsu, A. Han, S.-F. Leung, J.-K. Huang, Y. Wan, S. Liu, J. Zhang, J.-H. He, W.-H. Chang, Z. L. Wang, X. Zhang, L.-J. Li, ACS Nano. 2018, 12, 4976-4983. 
[15] M. Dai, Z. Wang, F. Wang, Y. Qiu, J. Zhang, C.-Y. Xu, T. Zhai, W. Cao, Y. Fu, D. Jia, Y. Zhou, P.-A. Hu, Nano Lett. 2019, 19, 5410-5416.

[16] S. Velasco-Lozano, M. Knez, F. López-Gallego, ACS Appl. Energy Mater. 2018, 1, 2032-2040.

[17] A. Gagrai, V. Mundlapati, D. Sahoo, H. Satapathy, H. Biswal, ChemistrySelect. 2016, 1, 4326-4331.

[18] S. K. Ghosh, D. Mandal, ACS Sustain. Chem. Eng. 2017, 5, 8836-8843.

[19] D. Kim, S. A. Han, J. H. Kim, J.-H. Lee, S.-W. Kim, S.-W. Lee, Adv. Mater. 2020, 32, 1906989.

[20] S. Guerin, A. Stapleton, D. Chovan, R. Mouras, M. Gleeson, C. McKeown, M. R. Noor, C. Silien, F. M. F. Rhen, A. L. Kholkin, N. Liu, T. Soulimane, S. A. M. Tofail, D. Thompson, Nat. Mater. 2018, 17, 180-186.

[21] A. Kholkin, N. Amdursky, I. Bdikin, E. Gazit, G. Rosenman, ACS Nano. 2010, 4, 610-614.

[22] W. E. Geiger, Organometallics. 2007, 26, 5738-5765.

[23] D. Schaarschmidt, H. Lang, Organometallics. 2013, 32, 5668-5704.

[24] M. Alfonso, A. Espinosa Ferao, A. Tárraga, P. Molina, Inorg. Chem. 2015, 54, 7461-7473.

[25] Y. Hong, J. W. Y. Lam, B. Z. Tang, Chem. Soc. Rev. 2011, 40, 5361-5388.

[26] J. D. Luo, Z. L. Xie, J. W. Y. Lam, L. Cheng, H. Y. Chen, C. F. Qiu, H. S. Kwok, X. W. Zhan, Y. Q. Liu, D. B. Zhu, B. Z. Tang, Chem. Comm. 2001, 1740-1741.

[27] J. Yang, Z. Chi, W. Zhu, B. Z. Tang, Z. Li, Sci. China Chem. 2019, 62, 1090-1098.

[28] M. Liu, Q. Wu, H. Shi, Z. An, W. Huang, Acta Chim Sinica. 2018, 76, 246-258.

[29] C. Wang, B. Xu, M. Li, Z. Chi, Y. Xie, Q. Li, Z. Li, Mater. Horizons. 2016, 3, 220-225.

[30] J. Yang, X. Gao, Z. Xie, Y. Gong, M. Fang, Q. Peng, Z. Chi, Z. Li, Angew. Chem. Int. Ed. 2017, 56, 1529915303.

[31] Z. Hu, Y. Li, M. Kang, M. Islam, M. Chen, J. Zhang, Y. Xiao, X. Feng, C. Redshaw, M. Zhang, Q. Chen, S. Xie, W. Jacky, B. Tang, EcoMat. 2020, 2, 1-12.

[32] S. Varughese, M. S. R. N. Kiran, U. Ramamurty, G. R. Desiraju, Chem Asian J. 2012, 7, 2118-2125.

[33] A. Worthy, A. Grosjean, M. C. Pfrunder, Y. Xu, C. Yan, G. Edwards, J. K. Clegg, J. C. McMurtrie, Nat. Chem. 2018, $10,65-69$.

[34] T. Vy, R. Soklaski, Y. Liang, L. Yang, Phys. Rev. B. 2014, 89, 235319.

[35] X. Wang, A. M. Jones, K. L. Seyler, T. Vy, Y. Jia, H. Zhao, H. Wang, L. Yang, X. Xu, F. Xia, Nat. Nanotechnol. 2015, 10, 517-521.

[36] C. Wang, H. Dong, L. Jiang, W. Hu, Chem. Soc. Rev. 2018, 47, 422-500. 
[37] G. Giri, E. Verploegen, S. C. B. Mannsfeld, S. Atahan-Evrenk, D. H. Kim, S. Y. Lee, H. A. Becerril, A. Aspuru-Guzik, M. F. Toney, Z. Bao, Nature. 2011, 480, 504-508.

[38] W. Wu, L. Wang, Y. Li, F. Zhang, L. Lin, S. Niu, D. Chenet, X. Zhang, Y. Hao, T. F. Heinz, J. Hone, Z. L. Wang, Nature. 2014, 514, 470-474.

[39] A. A. Shevyrin, A. G. Pogosov, A. K. Bakarov, A. A. Shklyaev, Phys. Rev. Lett. 2016, 117, 017702.

[40] T. Jia, H.-R. Fuh, D. Chen, M. Abid, M. Abid, D. Zhang, A. B. Sarker, J. Cho, M. Choi, B. S. Chun, H. Xu, C. O. Coileain, H. Liu, C.-R. Chang, H.-C. Wu, Adv. Electron. Mater. 2018, 4, 1700447.

[41] D. Zhang, Z. Yang, P. Li, M. Pang, Q. Xue, Nano Energy. 2019, 65, 103974.

[42] H. Zhang, Z. Zhao, A. T. Turley, L. Wang, P. R. McGonigal, Y. Tu, Y. Li, Z. Wang, R. T. K. Kwok, J. W. Y. Lam, B. Z. Tang, Adv. Mater. 2020, 32, 2001457.

[43] Y.-B. Gong, P. Zhang, Y.-r. Gu, J.-Q. Wang, M.-M. Han, C. Chen, X.-J. Zhan, Z.-L. Xie, B. Zou, Q. Peng, Z.G. Chi, Z. Li, Adv. Opt. Mater. 2018, 6, 1800198.

[44] J.-L. Do, T. Friščić, ACS Cent. Sci. 2017, 3, 13-19.

[45] J. G. Hernández, C. Bolm, J. Org. Chem. 2017, 82, 4007-4019.

[46] A. Tuer, S. Krouglov, R. Cisek, D. Tokarz, V. Barzda, J Comput. Chem. 2011, 32, 1128-1134.

[47] C. Adamo, V. Barone, J. Chem. Phys. 1999, 110, 6158-6170.

[48] I. Engeler, H. J. Hendricks Franssen, R. Müller, F. Stauffer, J. Hydrol. 2011, 397, 295-305.

[49] A. Aditya Prasad, K. Muthu, V. Meenatchi, M. Rajasekar, R. Agilandeshwari, K. Meena, J. Vijila Manonmoni, S. P. Meenakshisundaram, Spectrochim Acta A. 2015, 140, 311-327. 